\title{
ON THE ASYMPTOTIC BEHAVIOR OF FUNCTIONS HARMONIC IN A DISC
}

\author{
J. E. MCMILLAN
}

Let $D$ be the open unit disc, and let $C$ be the unit circle in the complex plane. Let $f$ be a (real-valued) function that is harmonic in $D$. A simple continuous curve $\beta: z(t)(0 \leqq t<1)$ contained in $D$ such that $|z(t)| \rightarrow 1$ as $t \rightarrow 1$ is a boundary path with end $\bar{\beta} \cap C$ (the bar denotes closure). If also $f(z(t)) \rightarrow a(-\infty \leqq a \leqq+\infty)$ as $t \rightarrow 1$, then $\beta$ is an asymptotic path of $f$ for the value $a$, and $f$ is said to have the asymptotic value $a$. If there is an asymptotic path of $f$, for a value $a(-\infty \leqq a \leqq+\infty)$, with end the point $\zeta$ of $C$, then $f$ is said to have the asymptotic value $a$ at $\zeta$. Let $A$ denote the set of points of $C$ at which $f$ has an asymptotic value. If $-\infty \leqq u<v \leqq+\infty$, set $A(u, v)=\{\zeta \in C$ : there exists a real number $a$ such that $f$ has the asymptotic value $a$ at $\zeta$ and $u<a<v\}$, and set $A^{\prime}=A(-\infty,+\infty)$. We use repeatedly the fact that $A(u, v)$ is a Borel set (see [5, Theorem 7 (iii)]), and is therefore measurable. Let $d(z, S)$ denote the Euclidean distance from the point $z$ to the set $S$ in the plane. For a sequence $\left\{\Gamma_{n}\right\}$ of Jordan $\operatorname{arcs}$ in $D$ and an $\operatorname{arc} r$ in $C$, the symbol $\Gamma_{n} \rightarrow \gamma$ means that to each $\varepsilon>0$ there corresponds a natural number $n_{\varepsilon}$ such that if $n>n_{\varepsilon}$, then

$$
\Gamma_{n} \subset\{z: d(z, \gamma)<\varepsilon\} \text { and } \gamma \subset\left\{z: d\left(z, \Gamma_{n}\right)<\varepsilon\right\} .
$$

The following theorem is closely related to the theorem [4, Theorem 1] for meromorphic functions.

TheOREM 1. Let $\zeta \in C$ and suppose that there exists a sequence $\left\{z_{n}\right\} \subset D$ such that $z_{n} \rightarrow \zeta$ and $f\left(z_{n}\right) \rightarrow+\infty$. Then at least one of the following three statements holds.

(i) Each open arc containing 5 contains the end of an asymptotic path of $f$ for the value $+\infty$.

(ii) $\zeta$ is one endpoint of an arc $r \subset C$ such that there exist a sequence $\left\{a_{n}\right\}$ of real numbers and a sequence $\left\{\Gamma_{n}\right\}$ of Jordan arcs such that

Received February 3, 1966. 


$$
a_{n} \uparrow+\infty, \Gamma_{n} \subset\left\{z \in D: f(z)=a_{n}\right\}, \Gamma_{n} \rightarrow \gamma .
$$

(iii) For each real number $M$ and open arc $\gamma$ containing $\zeta$, the set $\gamma \cap A(M,+\infty)$ has positive Lebesgue measure.

Remarks. At each $\zeta \in C$, the real part of the elliptic modular function satisfies neither (ii) nor (iii). The real part of a holomorphic function having the spiral asymptotic values 0 and 1 satisfies, at each $\zeta \in C$, neither (i) nor (iii). The real part $f$ of a holomorphic function constructed by MacLane [2, p. 75] is such that $A^{\prime}$ is dense on $C, f$ has neither of the asymptotic values $\pm \infty$, and there exists a set $E \subset C$ with positive measure such that $f$ does not have an asymptotic value at any point of $E$. Let $\zeta$ be a point of $C$ such that the intersection of $E$ with each open arc containing $\zeta$ has positive measure. Then neither (i) nor (ii) holds, and for each open arc $r$ containing $\zeta$, the measure of $\gamma \cap A^{\prime}$ is less than the measure of $\gamma$.

We first prove

Lemma. Let $\lambda$ be a real number, and suppose that $\Delta$ is a component of $\{z \in D: f(z)>\lambda\}$. Then either there exists an asymptotic path $\alpha$ of $f$ for the value $+\infty$ such that $\alpha \subset \Delta$, or there exists a set $E \subset C$ with positive exterior Lebesgue measure such that each $e^{i \theta} \in E$ is the end of an asymptotic path $\alpha_{\theta}$, for a finite value, such that $\alpha_{\theta} \subset \Delta$.

Remark 1. By making simple modifications in the following proof, an analogous lemma for holomorphic functions can be established. Thus, the proofs of the theorems [3, Theorem 2] and [4, Theorem 1], which are based on the lemma $[3$, Lemma 2$]$, can be simplified.

Remark 2. The proof of this lemma involves a combination of ideas from the papers [1], [2] and [3].

Proof of the lemma. Suppose that there does not exist an asymptotic path $\alpha$ of $f$ for the value $+\infty$ such that $\alpha \subset \Delta$. We prove that there exist a (finite) number $\lambda^{\prime} \geqq \lambda$ and a component $\Delta^{\prime}$ of $\left\{z \in \Delta: f(z)>\lambda^{\prime}\right\}$ such that $f$ is bounded in $\Delta^{\prime}$. If this were not the case, we could choose $\lambda_{n} \uparrow+\infty\left(\lambda_{n}>\lambda\right)$, let $\Delta_{1}$ be a component of $\left\{z \in \Delta: f(z)>\lambda_{1}\right\}$, let $\Delta_{2}$ be a component of $\left\{z \in \Delta_{1}: f(z)>\lambda_{2}\right\}$, and in this way define a sequence $\left\{\Delta_{n}\right\}$ such that $\Delta_{n+1}$ is a component of $\left\{z \in \Delta_{n}: f(z)>\lambda_{n+1}\right\}$. Let $\alpha: z(t)(0 \leqq t<1)$ be a boundary path that is 
eventually in each $\Delta_{n}$; that is, to each $n$ there corresponds $t_{n}\left(0 \leqq t_{n}<1\right)$ such that $z(t) \in \Delta_{n}$ if $t_{n}<t<1$. Since $\alpha$ is an asymptotic path of $f$ for the value $+\infty$ and $a \subset \Delta$, we have a contradiction; and we have established the existence of $\lambda^{\prime}$ and $d^{\prime}$ with the stated properties.

By the minimum principle, $\Delta^{\prime}$ is simply connected. Let $D_{w}=\{|w|<1\}$, and let $\varphi$ be a conformal mapping of $D_{w}$ onto $\Delta^{\prime}$. Set $F(w)=f(\varphi(w))\left(w \in D_{w}\right)$. The radial limit $F\left(e^{j \theta}\right)$ of the bounded harmonic function $F$ at $e^{i \theta}$ exists for almost all $e^{i n}$, and $F$ has a Poisson integral representation in terms of the radial limits $F\left(e^{i \theta}\right)$. Since $F(w)>\lambda^{\prime}\left(w \in D_{w}\right)$, there exists a set $E_{w}^{\prime} \subset\{|w|=1\}$, with positive measure, such that $F\left(e^{i \theta}\right)>\lambda^{\prime}$ if $e^{i \theta} \in E_{w v}^{\prime}$. Let $E_{t v}$ be a subset, with positive measure, of $E_{: y}^{\prime}$ such that the radial limit $\varphi\left(e^{i \theta}\right)$ of $\varphi$ at $e^{i \text { in }}$ exists for each $e^{i \theta} \in E_{w}$. If $\varphi\left(e^{i,}\right) \in D$, then $F\left(e^{i \theta}\right)=f\left(\varphi\left(e^{i \theta}\right)\right)=\lambda^{\prime}$, so $\varphi\left(e^{i \theta}\right) \in C$ if $e^{i 0} \in E_{w}$. Set $E_{z}=\left\{\varphi\left(e^{i n}\right): e^{i \theta} \in E_{w}\right\}$. By an extension of Löwner's lemma [6, p. 34], $E_{z}$ has positive exterior measure. But if $\zeta \in E_{z}, \zeta=\varphi\left(e^{i \cap}\right)$, the set $\left\{\varphi\left(r e^{i \theta}\right): 0 \leqq r<1\right\}$ is a boundary path, with end $\zeta$, that is contained in $\Delta^{\prime}$ and on which $f$ has the limit $F\left(e^{i \theta}\right)$ at $\zeta$. This completes the proof of the lemma.

Proof of Theorem 1. We clearly may suppose that $f(0)<f\left(z_{n}\right)-1(n \geqq 1)$. Let $A_{n}$ be the component of $\left\{z \in D: f(z)>f\left(z_{n}\right)-1\right\}$ that contains $z_{n}$. Since $f\left(z_{n}\right) \rightarrow+\infty$, each disc $\{|z| \leqq r\}(0<r<1)$ intersects only finitely many $\Delta_{n}$. Since $0 \neq \Delta_{n}$, there exists a level curve $L_{n}$ on the boundary of $\Delta_{n}$ such that 0 and $\Delta_{n}$ are contained in different components of $D-L_{n}$. Thus, it is easy to see that if (ii) does not hold, then the diameter of $\Delta_{n}$ tends to zero as $n \rightarrow \infty$. We suppose now that neither (i) nor (ii) holds. We wish to prove that (iii) holds, and we let $M$ be a real number and $r$ an open arc containing $\zeta$. Let $\gamma^{\prime}$ be an open subarc of $r$ that contains $\zeta$ and is such that no asymptotic path of $f$ for the value $+\infty$ has end contained in $\gamma^{\prime}$. Let $n_{0}$ be such that $f\left(z_{n_{0}}\right)-1>M$ and $\bar{\triangle}_{n_{0}} \cap C \subset \gamma^{\prime}$. It follows from the lemma that $r \cap A(M,+\infty)$ has positive exterior measure. Thus, since $A(M,+\infty)$ is measurable, (iii) holds; and the proof of Theorem 1 is complete.

It is well known that if $f$ is bounded above in a neighborhood of the point $\zeta$ of $C$, then $f$ has finite radial limits at almost all points of some open arc containing 5 . Thus, as a consequence of Theorem 1 we have

Corollary. Let $\gamma$ be an arc in $C$. Suppose that there exists a set $S \subset \gamma$, that is dense on $\gamma$ (i.e. $\gamma \subset \bar{S}$ ), such that to each $\zeta \in S$ there corresponds a boundary 
path with end $\zeta$ on which $f$ is bounded above. Then for each subarc $\gamma^{\prime}$ of $r$, either $f$ has the asymptotic value $+\infty$ at a point of $\gamma^{\prime}$, or $r^{\prime} \cap A^{\prime}$ has positive measure.

As a simple application of Theorem 1, we prove

ThEOREM 2. Let $\gamma$ be an arc in C. Suppose that there exists a set $S \subset \gamma$, that is dense on $r$, such that to each $\zeta \in S$ there corresponds a boindary path with end $\zeta$ on which $f$ is either bounded above or bounded below. Then for each subarc $\gamma^{\prime}$ of $r$, either $f$ has an infinite asymptotic value at a point of $r^{\prime}$, or $r^{\prime} \cap A^{\prime}$ has positive measure. In particular, $A$ is dense on $r$.

Remark. This result is closely related to theorems of MacLane [2, pp. 10, 25] for holomorphic functions.

Proof of Theorem 2. Suppose that there exists a subarc $\gamma^{\prime}$ of $r$ such that $f$ does not have an infinite asymptotic value at a point of $\gamma^{\prime}$, and $\gamma^{\prime} \cap A^{\prime}$ does not have positive measure. Then, since $A^{\prime}$ is measurable, $r^{\prime} \cap A^{\prime}$ has measure zero. Let $\zeta$ be an interior point of $\gamma^{\prime}$ and apply Theorem 1. Either there exists an asymptotic path of $f$ for the value $+\infty$ whose end is a subarc of $r^{\prime}$, or (ii) holds. In either case there exist a sequence $\left\{\Gamma_{n}\right\}$ of Jordan $\operatorname{arcs}$ in $D$ and a subarc $\gamma_{0}$ of $\gamma^{\prime}$ such that $\Gamma_{n} \rightarrow \gamma_{0}$ and the minimum value of $f$ on $\Gamma_{n}$ tends to $+\infty$ as $n \rightarrow \infty$. Now let $\zeta$ be an interior point of $r_{0}$ and apply Theorem 1 to the function $-f$. It follows as before that there exist a sequence $\left\{\Gamma_{n}^{\prime}\right\}$ of Jordan arcs in $D$ and a subarc $\gamma_{1}$ of $\gamma_{0}$ such that $\Gamma_{n}^{\prime} \rightarrow \gamma_{1}$ and the maximum value of $f$ on $\Gamma_{n}^{\prime}$ tends to $-\infty$ as $n \rightarrow \infty$. With this contradiction the proof of Theorem 2 is complete.

Similarly, we obtain

Theorem 3. Let $r$ be an arc in C. Suppose that there exists a set $S \subset \gamma$, that is dense on $\gamma$, such that to each $\zeta \in S$ there corresponds a boundary path with end $\boldsymbol{\zeta}$ on which $f$ is bounded. Then for each subarc $\gamma^{\prime}$ of $r$, either $f$ has both of the asymptotic values $+\infty$ and $-\infty$ at points of $r^{\prime}$, or $r^{\prime} \cap A^{\prime}$ has positive measure.

The following global result is an immediate consequence of the lemma.

THEOREM 4. Suppose that $f$ is not bounded above. Then either $f$ has the asymptotic value $+\infty$, or for each real number $M$, the set $A(M,+\infty)$ has positive measure. 
Remark. The real part $f$ of a holomorphic function constructed by MacLane $[2$, p. 71$]$ is such that $f$ has neither of the asymptotic values $\pm \infty$, and at each $\zeta \in C$,

$$
\underset{z \rightarrow \zeta}{\lim \sup } f(z)=+\infty \text { and } \liminf _{z \rightarrow \zeta} f(z)=-\infty .
$$

It is now easy to see that we also have

TheOREM 5. Either $f$ has both $+\infty$ and $-\infty$ as asymptotic values, or $A^{\prime}$ has positive measure.

\section{REFERENCES}

[1] F. Bagemihl and W. Seidel: Koebe arcs and Fatou points of normal functions, Comment. Math. Helv., 36 (1961), 9-18.

[2] G. R. MacLane: Asymptotic values of holomorphic functions, Rice Univ. Studies, 49 (1963), 1-83.

[3] J. E. McMillan: Asymptotic values of functions holomorphic in the unit disc, Michigan Math. J., 12 (1965), 141-154.

[4] J. E. McMillan: On local asymptotic properties, the asymptotic value sets, and ambiguous properties of functions meromorphic in the open unit disc, Ann. Acad. Sci. Fenn., A. I., 384 (1965), 1-12.

[5] J. E. McMillan: Boundary properties of functions continuous in a disc, Michigan Math. J. ( to appear).

[6] K. Noshiro: Cluster sets, Berlin-Göttingen-Heidelberg, 1960.

University of Wisconsin-Milwaukee 